J. Jpn. Soc. Comp. Statist., 20(2007), 1-18

\title{
EVALUATION OF STATISTICAL METHODS FOR ANALYSIS OF SMALL-SAMPLE LONGITUDINAL CLINICAL TRIALS WITH DROPOUTS
}

\author{
Takayuki Abe* and Manabu Iwasaki ${ }^{\dagger}$
}

\begin{abstract}
In longitudinal clinical trials that compare treatments of chronic diseases missing data occur mainly because of dropouts, where patients stop participating in the trial before the completion due to various reasons. Such incomplete data are often analyzed by using so-called completer analysis and/or LOCF (Last Observation Carried Forward). However, such procedures require strong assumptions for their validity. Multiple imputation (MI) (Rubin, 1987) is a valid method under MAR (Missing At Random). This method consists of three steps ("imputation", "analysis" and "combination") and various methods for MI also have been proposed. In this paper, we evaluate the performance of four methods for MI contrasted with completer analysis and LOCF via Monte-Carlo simulations in the context of small-sample longitudinal clinical trials for comparison of two treatments. The performance of these methods with non-normal data (i.e. mixture of responders and non-responders) is also examined.
\end{abstract}

\section{Introduction}

In order to compare treatments of chronic diseases, longitudinal clinical trials are typically conducted to collect data over time, where length of time can be a few weeks, several months, or even years. In these trials, it is frequently the case that patients drop out of a trial before completion due to various reasons, such as occurrence of adverse reactions, lack of efficacy, accidental events, and so on. Thus this leads to incomplete data. The dropout problem can be viewed as a special case of the missing data problem. In such situations researchers should consider the way to minimize difficulties due to incomplete data, i.e. bias in estimation and loss of information. For literature mainly devoted to analyses of incomplete data, see, for example, Iwasaki (2002a), Little and Rubin (2002), Schafer (1997) and Watanabe and Yamaguchi $(2000,2004)$. Recently, several commercial software packages have become available to handle incomplete data problems, and are used in various research areas such as medical and pharmaceutical studies. Comparisons of recent statistical methods for incomplete data and their software packages are reported by Abe, Inaba and Iwasaki (2005), Allison (2000) and Horton and Lipsitz (2001) among others. However, in practice, longitudinal incomplete data from clinical trials are still analyzed by completer analysis, which uses data from only patients who complete the trial, and/or last observation carried

\footnotetext{
*Biostatistics \& Research Decision Sciences, Banyu Pharmaceutical Co., Ltd., Kitanomaru Square, 1-13-12 Kudan-kita, Chiyoda-ku, Tokyo 102-8667, Japan

E-mail: takayuki_abe@merck.com

${ }^{\dagger}$ Department of Computer and Information Science, Seikei University, 3-3-1 Kichijoji-kitamachi, Musashinoshi, Tokyo 180-8633, Japan

E-mail: iwasaki@st.seikei.ac.jp

Key words: Missing data; Multiple imputation; Bayesian least squares; Predictive mean matching; LOCF
} 
forward (LOCF), which takes the last observed value prior to dropout from patients and these data as their final data. Comparisons of these classical techniques with recent computer intensive methods such as multiple imputation (MI) have not yet been fully made, particularly for relatively small sample-size cases.

In the context of longitudinal clinical trials with dropouts, some researchers, e.g. Barnes, Lindborg and Seaman (2006), Liu and Gould (2002) and Tang et al. (2005) have investigated the performance of MI. Liu and Gould (2002) evaluated a Bayesian least squares method, which is an imputation method in MI under various settings in a controlled longitudinal clinical trial to compare two treatments. Tang et al. (2005) discussed imputation methods (least squares and hot deck method) for a relatively large-sized clinical trial. Barnes et al. (2006) evaluated various imputation methods to estimate mean change from baseline within a treatment group in small sample longitudinal clinical trials $(n=20$ to 50$)$. However, validity of some imputation methods in MI in the context of relatively small-sample longitudinal controlled clinical trials to compare treatments has not been well examined. Therefore, in this paper, we evaluate four imputation methods, i.e. Bayesian least squares, predictive mean matching, propensity score and Markov Chain Monte Carlo in contrasting with completer analysis and LOCF using Monte Carlo simulations, in the analysis of FEV 1.0 data from a clinical trial to compare two treatments (placebo and new drug) for anti bronchial asthma. The comparison of the two treatments is the most typical case in clinical trials. The biases of matching-based imputation methods in the case of small sample are presented. All simulations were conducted by $\mathrm{SAS}^{\circledR}$ (version 9, MI procedure). Performance of the methods under multivariate normal (MVN) distributions with various covariance structures is examined because it is anticipated that the performance differs depending on the covariance structures. Finally performance of MVN-based methods under non-normal data (e.g. a mixture of responders and non-responders) is also examined.

This paper is structured as follows: Section 2 contains some preliminaries and notation. Statistical analysis methods examined in this paper are reviewed in section 3 . In section 4 , the biases in estimators from completer analysis and LOCF under MAR will be mathematically calculated, which will be used to examine the results of simulation in sections 5 and 6 . Sections 5 and 6 give simulation studies with various covariance structures and distributions. Section 7 is devoted to conclusion and some discussions.

\section{Some preliminaries and notation}

Let $Y_{i j k}$ denote the response of patient $i\left(i=1, \ldots, n_{k}\right)$ at visit $j(j=1, \ldots, T)$ in treatment $k$ (0: placebo, 1: new drug), where $n_{k}$ is the total number of patients in treatment $k$ and $T$ is the total number of visits. Then in this paper we consider the model

$$
Y_{i j k}=\mu_{0}+\pi_{j}+\tau_{k}+(\pi \tau)_{j k}+e_{i j k},
$$

where $\mu_{0}$ is the overall mean, $\pi_{j}$ is the main effect of visit $j, \tau_{k}$ is the main effect of treatment $k,(\pi \tau)_{j k}$ is the combination effect of visit $j$ and treatment $k$ and $e_{i j k}$ is the random error which follows an identical normal distribution. In section 5 we will assume four marginal covariance structures (i.e., Compound symmetry, AR (1) and so on) for the error term.

We introduce $R_{i j k}$ as a binary missing indicator variable (0: missing, 1: observed) corresponding to $Y_{i j k}$. The distribution of $R_{i j k}$ is assumed to be indexed by a parameter (vector) $\gamma$. The missingness pattern considered in this paper is assumed to be monotone, since non-responses mainly occur in actual clinical trials as dropouts from the trial, and which yield monotone patterns after rearrangement of patients in the dataset. Specifically, 
it is said that the missingness pattern is monotone if a response value of a patient at a visit is missing then the responses of the same patient after that visit are also missing. For monotone missing data, estimators of parameters can be derived explicitly (Little and Rubin, 2002). However the estimation of the standard error is still controversial specifically in small samples. Next, the missing mechanism is defined as follows. Let $Y_{o b s}$ be the observed components and $Y_{m i s}$ be the unobserved components in the dataset $Y$. Then Rubin (1976) classified the missing mechanism into the following three categories in terms of the conditional distribution of $R$ denoted by $p(R \mid Y, \gamma)$.
1) MCAR (Missing Completely At Random)
2) MAR (Missing At Random)
3) NMAR (Not Missing At Random)

$$
\begin{aligned}
& : p(R \mid Y, \gamma)=p(R \mid \gamma) \\
& : p(R \mid Y, \gamma)=p\left(R \mid Y_{\text {obs }}, \gamma\right) \\
& : p(R \mid Y, \gamma)=p\left(R \mid Y_{\text {obs }}, Y_{\text {mis }}, \gamma\right)
\end{aligned}
$$

In this paper we assume MCAR and MAR as the missing mechanisms. The reason is that, as Liu and Gould (2002) also referred to, the assumption of MAR (missing is conditionally independent given $Y_{o b s}$ ) is often plausible in actual clinical trials if we collect post-withdrawal information of patients as much as possible and include relevant variables in the imputation model.

\section{Statistical analysis for incomplete data}

\subsection{Completer analysis}

Completer analysis is an analysis based only on the patients with all pre-designed response values. It is also called complete case (CC) analysis or case deletion method. In the context of longitudinal clinical trials, this method includes only patients who have fully observed response at all visits in the trial. The completer analysis is valid if the missing mechanism is MCAR because the observed data are considered as a random sample from the complete data.

\subsection{Last observation carried forward (LOCF)}

In LOCF, all the missing responses of a patient after the last visit are imputed by the actually observed last value. Intuitively LOCF needs an assumption that the response profile is stable at and after the last visit. A difficulty in LOCF is that the standard error of the parameter estimate would be underestimated because it does not account for the variability of the observations after the missing point.

\subsection{Multiple imputation (MI)}

MI proposed by Rubin (1987) is a flexible analysis method, applicable to various incomplete data settings. It consists of three steps, "imputation", "analysis" and "combination" and is a simulation-based method motivated by Bayesian theory that performs statistical inference by imputing multiple draws (usually a small number of imputations (e.g. five) is sufficient with a moderate fraction of missing data (Rubin, 1987)) from the posterior predictive distribution of missing values. An advantage of MI is to prevent underestimation of the standard error of an estimate by taking into account the uncertainty of the imputations. Many imputation methods are proposed depending on how to generate the posterior predictive distribution of missing values. In section 3.3.1 four imputation methods in MI are summarized. Section 3.3.2 gives a brief summary of the combination step in MI. 


\subsubsection{Imputation step}

Four imputation methods in MI, Bayesian least squares, predictive mean matching, propensity score, and Markov Chain Monte Carlo, which will be evaluated in this paper are outlined below. For more details of the methods, see Rubin (1987) and SAS Institute Inc. (2004)

\section{1) Bayesian least squares (BLS)}

To impute the missing responses $Y_{i, J, k}$ at visit $J$, the following regression analysis of $Y_{i, J, k}$ on the all responses $Y_{i, j, k}(j=1, \ldots, J-1)$ observed at previous visits is performed for fully observed patients from visit 1 to $J$ by each treatment group.

$$
Y_{i, J, k}=\beta_{0, k}+\beta_{1, k} Y_{i, 1, k}+\beta_{2, k} Y_{i, 2, k}+\cdots+\beta_{J-1, k} Y_{i, J-1, k}+e_{i, J, k},
$$

where $\boldsymbol{\beta}_{k}=\left(\beta_{0, k}, \beta_{1, k}, \beta_{2, k}, \ldots, \beta_{J-1, k}\right)$ is the regression parameter vector and $e_{i, J, k}$ is the error term, which follows $N\left(0, \sigma_{k}^{2}\right)$.

Posterior distributions of the parameters (regression parameters $\boldsymbol{\beta}_{k}$ and error variance $\sigma_{k}^{2}$ ) are derived by using the parameter estimates obtained from the complete-case regression analysis. For each imputation, parameters are simulated from their posterior distributions, and the missing values at the visit are then replaced by a random drawing from the posterior predictive distribution of $Y_{i, J, k}$. This procedure is repeated sequentially for each visit to create one set of completed data. The whole procedure is repeated $m$ times independently to yield $m$ imputations by each treatment group.

\section{2) Predictive mean matching (PMM)}

PMM (Heitjan and Little, 1991) is closely related to the above mentioned BLS. The only difference between the two procedures lies in the method in selecting imputations based on the predicted values for missing values from regression analysis. BLS creates imputations for missing values based on random draws from the posterior predictive distribution of the missing values, whilst PMM finds $k_{0}$ candidates with the closest predicted responses and creates imputations randomly from the candidates' actual observed response in place of the missing value of the non-respondent. An advantage of PMM is that all imputations are the responses actually observed. On the other hand, a disadvantage is that it is sometimes difficult to find candidates which have really close responses for small-sample data. In this paper, $k_{0}=5$ is used as the number of candidates.

\section{3) Propensity score (PS)}

PS (Lavori, Dawson and Shera, 1995) is a nonparametric method to generate imputations. First, propensity scores (Rosenbaum and Rubin, 1983) are estimated by a logistic regression with missing indicator variable $R_{i J k}$ as the dependent variable and with responses $Y_{i j k}(j=1, \ldots, J-1)$ observed at previous visits as explanatory variables. Second, each patient is classified into one of $k$ strata depending on its propensity score and $n_{0}$ patients are randomly drawn with replacement from $n_{0}$ patients with response observed in the stratum. Within every stratum, imputations for non-respondents are randomly drawn from the $n_{0}$ patients also with replacement. This procedure is referred to as the approximate Bayesian bootstrap (ABB). The procedure above mentioned is repeated sequentially for each visit to create completed data. These procedures are repeated $m$ times independently and create $m$ imputations for each treatment group. In this paper, $k=4$ is adopted to handle smallsample data because a stratum with the highest propensity score may have no actually 
observed values in the case of a small sample.

\section{4) Markov chain monte carlo (MCMC)}

MCMC is a collection of techniques for creating pseudorandom draws from a distribution of interest. Data Augmentation (Tanner and Wong, 1987) is a form of MCMC and consists of two steps, i.e. imputation step (I-step) and posterior step (P-step). Repetition of these two steps creates Markov chains of sets of parameters and missing values. MCMC provided by $\mathrm{SAS}^{\circledR}$ version 9 (PROC MI) is based on data augmentation with a single chain and takes 200 burn-in iterations before the first imputation and 100 iterations between imputations. The EM algorithm (Dempster, Laird and Rubin, 1977) is used to compute the posterior mode with a noninformative prior as the starting value for the chain. In the I-step, missing values are randomly drawn from the conditional distribution of the missing response given the parameters. In the $\mathrm{P}$-step, parameters are randomly drawn from the conditional distribution of the parameters given the missing values.

\subsubsection{Combination step}

Let $\hat{\theta}^{(l)}=\hat{\theta}\left(Y_{o b s}, Y_{m i s}^{(l)}\right)$ and $U^{(l)}=U\left(Y_{o b s}, Y_{m i s}^{(l)}\right)(l=1, \ldots, m)$ denote an estimate of $\theta$ and its estimated variance. Then the overall estimate is $\bar{\theta}$ given by

$$
\bar{\theta}=m^{-1} \sum_{l}^{m} \hat{\theta}^{(l)} .
$$

The sampling variance $T$ of $\bar{\theta}$ is estimated by the weighted sum

$$
T=\left(1+m^{-1}\right) B+\bar{U}
$$

of the within-imputation variance $\bar{U}=m^{-1} \sum_{l}^{m} U^{(l)}$ and the between-imputation variance

$$
B=(m-1)^{-1} \sum_{l}^{m}\left(\hat{\theta}^{(l)}-\bar{\theta}\right)^{2} .
$$

Then statistical inferences about $\theta$ will be performed based on the student's $t$-approximation

$$
(\bar{\theta}-\theta) / \sqrt{T} \sim t_{v}
$$

with $\nu$ degrees of freedom, where

$$
\nu=(m-1)\left[1+\frac{\bar{U}}{\left(1+m^{-1}\right) B}\right]^{2} .
$$

Barnard and Rubin (1999) proposed modified degrees of freedom for small samples given by

$$
\nu_{m}^{*}=\left[\frac{1}{\nu}+\frac{1}{\hat{\nu}_{o b s}}\right]^{-1},
$$

where $\hat{\nu}_{o b s}=(1-\gamma) \nu_{0}\left(\nu_{0}+1\right) /\left(\nu_{0}+3\right), \gamma=\left(1+m^{-1}\right) B / T$ and $\nu_{0}$ is the degrees of freedom for complete data. In this paper, the modified formula of the degrees of freedom (5) is adopted. 


\section{Evaluation of the bias in completer analysis and LOCF}

In this section, the biases in the estimates of a treatment effect defined as the change from baseline obtained from completer analysis and LOCF will be mathematically evaluated in the context of the longitudinal data with dropout under the MAR assumption. Although the simulation studies in section 5 assume a 4-variate normal distribution corresponding to four time points, the biases under a trivariate normal distribution are evaluated in this section. The reasons are 1) expectations of biases can not be explicitly derived under the simulation design in section 5 with 4 -variate normal. 2) The results from the trivariate normal can be treated as an approximation to the 4 -variate normal results because the missing pattern (Figure 1 (a)) where the trivariate results are applicable is not so different from that (Figure 1 (b)) assumed in the simulation studies in section 5 .

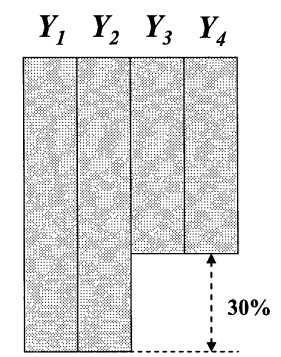

(a) A missing pattern assumed in section 4

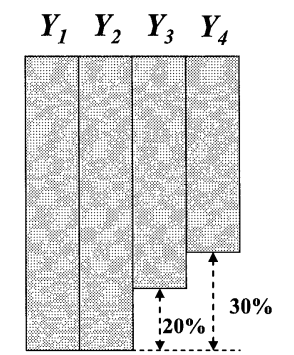

(b) A missing pattern in section 5

Fig. 1: Two missing patterns

It is assumed that a response vector $\boldsymbol{Y}=\left(Y_{1}, Y_{2}, Y_{3}\right)^{T}$ measured at three visits consisting of one baseline and two visits during the treatment-period follows a trivariate normal distribution with mean vector $\boldsymbol{\mu}=\left(\mu_{1}, \mu_{2}, \mu_{3}\right)^{T}$ and the covariance matrix

$$
\sum=\left(\begin{array}{ccc}
\sigma_{1}^{2} & \rho_{12} \sigma_{1} \sigma_{2} & \rho_{13} \sigma_{1} \sigma_{3} \\
\rho_{12} \sigma_{1} \sigma_{2} & \sigma_{2}^{2} & \rho_{23} \sigma_{2} \sigma_{3} \\
\rho_{13} \sigma_{1} \sigma_{3} & \rho_{23} \sigma_{2} \sigma_{3} & \sigma_{3}^{2}
\end{array}\right) .
$$

Let us consider the situation that our primary measure of interest is the change from baseline $Y_{3}-Y_{1}$ and that missing occurs in variable $Y_{3}$ only. The missing mechanism is assumed to be the left censoring that depends on $Y_{2}$. Specifically $Y_{3}$ will be missing if $Y_{2}<a$ for a certain constant $a$. This missing mechanism is MAR because a missing at the third visit depends on the observed value at the second visit. To evaluate the biases in the estimates of the change from baseline obtained from completer analysis and LOCF, conditional distributions of a trivariate normal distribution will be derived.

The conditional expectation of $Y_{2}, E\left[Y_{2} \mid Y_{2} \geq a\right]$ is given by

$$
\mu_{2}^{\prime}=E\left[Y_{2} \mid Y_{2} \geq a\right]=\mu_{2}+\frac{\varphi(c)}{1-\Phi(c)} \sigma_{2}
$$

where $c=\left(a-\mu_{2}\right) / \sigma_{2}$, and $\varphi(\cdot)$ and $\Phi(\cdot)$ respectively denote the probability density function and the cumulative distribution function of the standard normal distribution. The 
conditional expectation of $Y_{3}, E\left[Y_{3} \mid Y_{2} \geq a\right]$ is given by

$$
\mu_{3}^{\prime}=E\left[Y_{3} \mid Y_{2} \geq a\right]=\mu_{3}+\frac{\sigma_{23}}{\sigma_{2}^{2}}\left(\mu_{2}^{\prime}-\mu_{2}\right) .
$$

Details of derivation of the above formulas can be found in Johnson and Kotz $(1970,1972)$ and Iwasaki (2002b).

\section{1) Completer analysis}

The expectation of the change from baseline based on completer analysis, $E\left[\left(Y_{3}-\right.\right.$ $\left.\left.Y_{1}\right)_{C C}\right]$, is

$$
\begin{aligned}
E\left[\left(Y_{3}-Y_{1}\right)_{C C}\right]=E\left[Y_{3}-Y_{1} \mid Y_{2} \geq a\right] & =\mu_{3}+\frac{\sigma_{23}}{\sigma_{2}^{2}}\left(\mu_{2}^{\prime}-\mu_{2}\right)-\left(\mu_{1}+\frac{\sigma_{12}}{\sigma_{2}^{2}}\left(\mu_{2}^{\prime}-\mu_{2}\right)\right) \\
& =\mu_{3}-\mu_{1}+\left(\frac{\sigma_{23}}{\sigma_{2}^{2}}-\frac{\sigma_{12}}{\sigma_{2}^{2}}\right)\left(\mu_{2}^{\prime}-\mu_{2}\right) \\
& =\mu_{3}-\mu_{1}+\left(\frac{\sigma_{23}}{\sigma_{2}^{2}}-\frac{\sigma_{12}}{\sigma_{2}^{2}}\right) \frac{\varphi(c)}{1-\Phi(c)} \sigma_{2} .
\end{aligned}
$$

From (6) it is observed that the bias in the estimate of change based on completer analysis is expressed by the product of the difference in regression coefficients and the bias in $\mu_{2}$ due to censoring. It follows that completer analysis gives an unbiased estimator if variances are the same among all visits and the covariance structure is compound symmetry.

\section{2) LOCF}

The expectation of the change from baseline based on LOCF, $E\left[\left(Y_{3}-Y_{1}\right)_{L O C F}\right]$, is given by

$$
\begin{aligned}
E\left[\left(Y_{3}-Y_{1}\right)_{L O C F}\right] & =(1-\pi) E\left[Y_{3} \mid Y_{2} \geq a\right]+\pi E\left[Y_{2} \mid Y_{2}<a\right]-\mu_{1} \\
& =(1-\pi)\left(\mu_{3}+\frac{\sigma_{23}}{\sigma_{2}^{2}}\left(\mu_{2}^{\prime}-\mu_{2}\right)\right)+\pi\left(\mu_{2}+\frac{-\varphi(c)}{\Phi(c)} \sigma_{2}\right)-\mu_{1} \\
& =\left((1-\pi) \mu_{3}+\pi \mu_{2}-\mu_{1}\right)+(1-\pi) \frac{\sigma_{23}}{\sigma_{2}^{2}}\left(\mu_{2}^{\prime}-\mu_{2}\right)-\varphi(c) \sigma_{2} \\
& =\left(\mu_{3}-\mu_{1}-\pi\left(\mu_{2}-\mu_{3}\right)\right)-\left(1-\frac{\sigma_{23}}{\sigma_{2}^{2}}\right) \varphi(c) \sigma_{2},
\end{aligned}
$$

where $\pi=\Phi(c)$ denotes the fraction of missing. Hence, the bias in the estimate of change from baseline based on LOCF is expressed by a function of the difference between $\mu_{2}$ and $\mu_{3}$, the correlation between $Y_{2}$ and $Y_{3}$, the fraction of missing, and the standard deviation of $Y_{2}$. The bias in the estimate based on LOCF approaches zero as the correlation approaches one under $\mu_{2}=\mu_{3}$ (The response reaches plateau status at visit 2) and the variance of $Y_{2}$ is the same as that of $Y_{3}$. Even in the case of the response profile being a plateau, estimates of the change from baseline based on LOCF have a negative bias proportional to the product of $(1-$ correlation $)$ and the standard deviation of $Y_{2}$.

\section{Simulation study I: Multivariate normal data}

In this section, simulation studies will be shown to evaluate the performance of six statistical methods for incomplete data described in section 3 (Completer analysis, LOCF, 
BLS, PMM, PS and MCMC) in the cases of multivariate normal (MVN) data. In the next section we also evaluate these methods in the case of a non-normal data structure, namely, a finite mixture of responders and non-responders which is a case frequently observed in actual clinical trials. In simulation studies the number of imputations in MI is set to five $(m=5)$.

\subsection{Simulation design}

\section{a) The data model}

As illustrated in Abe et al. (2005), our simulation design is based on FEV1.0 data, which is one of the endpoints used in clinical trials for therapeutic agents against bronchial asthma. FEV1.0 is one of pulmonary function tests to measure the lung function and represents the volume of expired air $(l)$ per second. All patients are randomly assigned to one of two treatment groups (a new drug or a placebo). The sample size is set to 100 for each group. FEV 1.0 measurements are obtained at four visits: i.e. one baseline visit and three subsequent treatment-period visits. It is assumed here that missing occurs in the response $Y_{j k}$ at visit $j(j=3$ or 4$)$ in treatment $k$ (0: placebo, 1: new drug) of the response vector $\boldsymbol{Y}_{k}=\left(Y_{1 k}, Y_{2 k}, Y_{3 k}, Y_{4 k}\right)^{T}$. The objective of this clinical trial is comparison of the change from baseline $\left(Y_{4 k}-Y_{1 k}\right)$ between treatment groups. The fractions of missing at visit 3 and 4 are assumed to be $20 \%$ and 30\%, respectively. The mean vectors of FEV 1.0 for new drug group and placebo are set to be $E\left[\boldsymbol{Y}_{1}\right]=(2.50,2.75,2.80,2.83)^{T}$ and $E\left[\boldsymbol{Y}_{0}\right]=(2.50,2.58,2.60,2.60)^{T}$, respectively. The plots of average FEV1.0 value at each visit are shown in Figure 2.

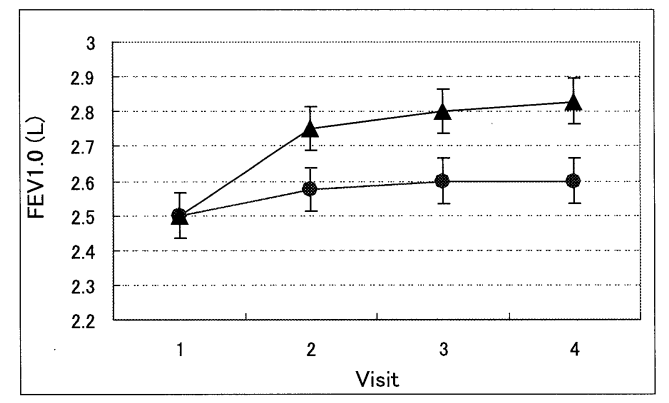

Fig. 2: Average profile in FEV1.0 (Mean $\pm \mathrm{SE})(\boldsymbol{\Lambda}$ : new drug, $\mathbf{O}$ : placebo)

It is assumed that the standard deviations of FEV 1.0 value at four visits are the same and set to 0.64 . The following four correlation matrices are considered and the changes in correlation coefficients in terms of distances between two time points for each correlation matrix are shown in Figure 3. The mean and standard deviation in FEV1.0 are determined based on the results from an actual clinical trial already conducted (Reiss et al., 1998).

In the simulation study, 100 random numbers that follow 4 -variate normal distributions with each mean vector and covariance matrix are generated by each treatment group. The number of replications in the simulation is 1000 . 


$$
\begin{aligned}
R_{C S(\text { Strong })} & =\left(\begin{array}{cccc}
1 & 0.80 & 0.80 & 0.80 \\
0.80 & 1 & 0.80 & 0.80 \\
0.80 & 0.80 & 1 & 0.80 \\
0.80 & 0.80 & 0.80 & 1
\end{array}\right), \quad R_{C S(\text { Weak })}=\left(\begin{array}{cccc}
1 & 0.20 & 0.20 & 0.20 \\
0.20 & 1 & 0.20 & 0.20 \\
0.20 & 0.20 & 1 & 0.20 \\
0.20 & 0.20 & 0.20 & 1
\end{array}\right), \\
R_{A R(1)} & =\left(\begin{array}{cccc}
1 & 0.80 & 0.64 & 0.51 \\
0.80 & 1 & 0.80 & 0.64 \\
0.64 & 0.80 & 1 & 0.80 \\
0.51 & 0.64 & 0.80 & 1
\end{array}\right), \quad R_{\text {Toeplitz }}=\left(\begin{array}{cccc}
1 & 0.80 & 0.40 & 0.20 \\
0.80 & 1 & 0.80 & 0.40 \\
0.40 & 0.80 & 1 & 0.80 \\
0.20 & 0.40 & 0.80 & 1
\end{array}\right) .
\end{aligned}
$$

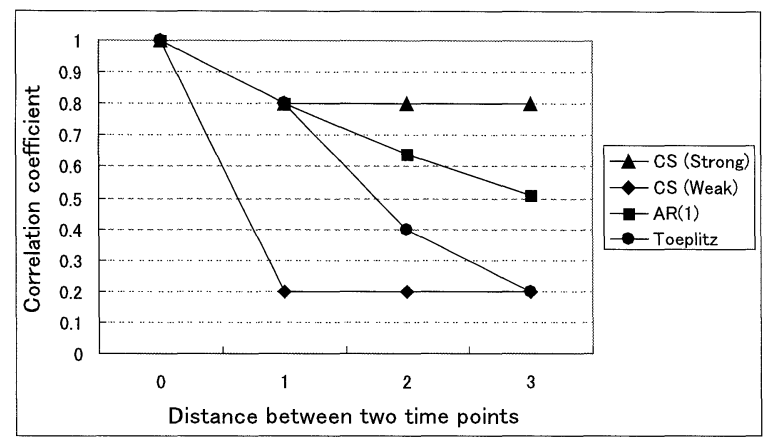

Fig. 3: Correlation coefficients and distances between two time points for each covariance matrix

\section{b) The missing mechanism model}

On the simulated FEV1.0 data missing values are generated using the following logistic models for MCAR and MAR respectively.

$$
\begin{aligned}
& \operatorname{MCAR}: \operatorname{logit}\left(p\left(R\left(Y_{j k}\right) \mid \alpha_{j(1)}\right)\right)=\alpha_{j(1)}, \\
& \operatorname{MAR}: \operatorname{logit}\left(p\left(R\left(Y_{j k}\right) \mid X_{j-1, k}, \alpha_{j(2)}, \beta\right)\right)=\alpha_{j(2)}+\beta X_{j-1, k},
\end{aligned}
$$

where $R\left(Y_{j k}\right)$ is the missing indicator variable for the response $Y_{j k}$ at visit $j(j=3,4)$ in treatment $k$ and $X_{j-1, k}$ is a standardized response of $Y_{j-1, k}$ to have zero mean and unit variance. If $\beta=0$, then the missing mechanism is MCAR. If the missing probability depends on the response at the previous visit then the mechanism becomes MAR. The parameters in the logistic model are set to $\beta=3$ and $\alpha_{j(l)}(l=1,2)$ being a constant to generate the fraction of missing values.

\section{c) Evaluation measures}

In the simulation studies 1000 sets of incomplete data are analyzed by six statistical methods (Completer analysis, LOCF, BLS, PMM, PS and MCMC) and this simulation study has two major parameters of interest: one is the mean change from baseline at the final visit $E\left[Y_{4 k}-Y_{1 k}\right]$ by each treatment group and the other is the between-group difference 
in the mean change, $E\left[\left(Y_{41}-Y_{11}\right)-\left(Y_{40}-Y_{10}\right)\right]$. Both parameters are estimated by using the above mentioned statistical methods.

In the simulation studies the standardized bias, the half length of the $95 \%$ confidence interval and the empirical coverage probability are used as three measures to evaluate the performance of each statistical method. The standardized bias of the parameter estimates (i.e. average change within each treatment group and between-group difference in the change) is defined as (point estimate - true parameter value)/SE. Standard errors (SEs) from MI are square root of the formula (2). In terms of the half length of the $95 \%$ confidence intervals for both parameters, the intervals are based on student's $t$-approximation and degrees of freedom (5) is used for four types of MI methods because of small-sample. The average for each measure across 1000 replications is presented. The empirical coverage probabilities $\hat{p}=x / 1000$ of the $95 \%$ confidence intervals are also shown, where $x$ is the number of times in which $95 \%$ confidence interval contains the true parameter value. In the case of 1000 replications the $\mathrm{SE}$ of the coverage probabilities is $\sqrt{0.95 \times 0.05 / 1000}=0.0069$, which is less than $0.7 \%$.

\subsection{Results}

The results for individual treatment groups from the simulation studies are summarized in Table 1 (a) to (d) corresponding to each covariance structure mentioned in the previous subsection. The results from the between-group difference in change (new drug - placebo) are summarized in Table 2 (a) and (b). The simulation results are summarized as the following.

a) MCAR

1) Standardized bias of mean change

(1) Within each treatment group

Only LOCF gave substantially biased estimators particularly in the new drug group whose response pattern was not plateau. In the placebo group whose response achieved plateau, the biases caused by LOCF were less than $10 \%$ of the standard error of their estimates.

\section{(2) Between-group difference in change}

Only LOCF had bias in the between-group difference in the change from baseline. The biases of the estimates by LOCF were less than $30 \%$ of the standard error.

\section{2) Coverage probability and interval length}

\section{(1) Within each treatment group}

For the length of the $95 \%$ confidence interval, LOCF provided the shortest intervals in both treatment groups for all covariance structures, which leads to overestimation of the precision of the estimates. In the new drug group, actual coverage probabilities of completer analysis and MI (except for PMM) are higher than the nominal level (two-sided 95\%). An actual coverage probability greater than $95 \%$ corresponds to smaller type I error rate than two-sided 5\% significance level in hypothesis testing. The actual coverage probabilities of PMM and LOCF were less than the nominal level by $2 \%$. In the placebo group where response achieved plateau, actual coverage probabilities were closer to the nominal level.

\section{(2) Between-group difference in change}

The confidence intervals of LOCF were shortest. For actual coverage probabilities, less than $2 \%$ reduction from the nominal level was observed in PMM, whilst the actual coverage 
Table 1: Standardized bias, half length of $95 \%$ CI and coverage probability for change from baseline at the final visit within treatment group ( $n=100$ / group)

(a) MVN (Covariance: Compound Symmetry (Strong))

\begin{tabular}{|c|c|c|c|c|c|c|}
\hline \multirow[b]{2}{*}{ Methods } & \multicolumn{3}{|c|}{ Treatment Group } & \multicolumn{3}{|c|}{ Placebo } \\
\hline & \multicolumn{2}{|c|}{$\begin{array}{c}\text { Standardized Bias } \\
(1 / 2 \text { of } 95 \% \mathrm{CI})\end{array}$} & \multirow[t]{2}{*}{$\begin{array}{c}\text { Coverage } \\
\text { Probability }(\%)\end{array}$} & \multicolumn{2}{|c|}{$\begin{array}{c}\text { Standardized Bias } \\
(1 / 2 \text { of } 95 \% \mathrm{CI})\end{array}$} & \multirow[t]{2}{*}{$\begin{array}{c}\text { Coverage } \\
\text { Probability }(\%)\end{array}$} \\
\hline \multicolumn{5}{|c|}{ Missing mechanism: MCAR } & & \\
\hline BLS & 0.03 & $(0.10)$ & 94.8 & -0.01 & $(0.10)$ & 95.0 \\
\hline PMM & 0.02 & $(0.09)$ & 93.7 & 0.00 & $(0.09)$ & 94.2 \\
\hline PS & 0.01 & $(0.15)$ & 99.3 & 0.00 & $(0.15)$ & 98.7 \\
\hline MCMC & 0.00 & $(0.10)$ & 94.2 & -0.02 & $(0.10)$ & 95.5 \\
\hline $\mathrm{CC}$ & 0.01 & $(0.10)$ & 94.8 & -0.02 & $(0.10)$ & 94.9 \\
\hline LOCF & -0.47 & $(0.08)$ & 92.6 & -0.09 & $(0.08)$ & 94.7 \\
\hline \multicolumn{7}{|c|}{ Missing mechanism: MAR } \\
\hline BLS & 0.01 & $(0.11)$ & 95.2 & 0.03 & $(0.11)$ & 95.3 \\
\hline PMM & 1.02 & $(0.10)$ & 80.5 & 1.01 & $(0.10)$ & 81.4 \\
\hline PS & 0.75 & $(0.13)$ & 89.2 & 0.73 & $(0.13)$ & 90.8 \\
\hline MCMC & 0.01 & $(0.11)$ & 95.6 & 0.00 & $(0.11)$ & 94.8 \\
\hline $\mathrm{CC}$ & 0.02 & $(0.10)$ & 96.2 & 0.00 & $(0.10)$ & 95.8 \\
\hline LOCF & -1.79 & $(0.08)$ & 58.7 & -1.46 & $(0.08)$ & 69.1 \\
\hline
\end{tabular}

(b) MVN (Covariance: Compound Symmetry (Weak))

\begin{tabular}{lcccccc}
\hline \multirow{2}{*}{ Methods } & \multicolumn{3}{c}{ Treatment Group } & \multicolumn{3}{c}{ Placebo } \\
\cline { 2 - 7 } & $\begin{array}{c}\text { Standardized Bias } \\
(1 / 2 \text { of } 95 \% \text { CI })\end{array}$ & $\begin{array}{c}\text { Coverage } \\
\text { Probability }(\%)\end{array}$ & $\begin{array}{c}\text { Standardized Bias } \\
(1 / 2 \text { of } 95 \% \text { CI })\end{array}$ & $\begin{array}{c}\text { Coverage } \\
\text { Probability }(\%)\end{array}$ \\
\hline Missing mechanism: MCAR & & & & & \\
BLS & 0.00 & $(0.19)$ & 94.9 & -0.02 & $(0.19)$ & 95.1 \\
PMM & 0.00 & $(0.18)$ & 93.0 & -0.03 & $(0.18)$ & 94.8 \\
PS & 0.00 & $(0.19)$ & 95.8 & -0.02 & $(0.19)$ & 94.5 \\
MCMC & 0.00 & $(0.19)$ & 94.7 & -0.03 & $(0.19)$ & 94.7 \\
CC & -0.03 & $(0.19)$ & 94.9 & -0.04 & $(0.19)$ & 94.0 \\
LOCF & -0.25 & $(0.16)$ & 93.7 & -0.06 & $(0.16)$ & 95.0 \\
\hline Missing mechanism:MAR & -1 & & & & & \\
\hline BLS & -0.01 & $(0.24)$ & 94.8 & -0.02 & $(0.23)$ & 95.6 \\
PMM & 0.29 & $(0.20)$ & 92.5 & 0.25 & $(0.20)$ & 93.8 \\
PS & 0.12 & $(0.23)$ & 93.7 & 0.06 & $(0.24)$ & 93.4 \\
MCMC & 0.00 & $(0.23)$ & 95.4 & -0.01 & $(0.23)$ & 95.2 \\
CC & -0.03 & $(0.20)$ & 94.5 & -0.02 & $(0.20)$ & 95.2 \\
LOCF & -3.02 & $(0.17)$ & 16.4 & -2.85 & $(0.17)$ & 21.6 \\
\hline
\end{tabular}

probabilities in other methods were close to the nominal one.

\section{b) MAR}

\section{1) Standardized bias of mean change}

\section{(1) Within each treatment group}

The biases of the estimates by LOCF were largest (1.5 to 3 times larger relative to the standard error) in all methods under all covariance structures for both treatment groups. As shown in (7), especially in the case of weak correlation matrix, bias was the largest. For matching-based methods (PMM and PS), biases in estimates were less than $102 \%$ and $77 \%$ of standard error, respectively. The reason seems to be that the number of matching 
Table 1 (cont.)

(c) MVN (Covariance: AR(1))

\begin{tabular}{|c|c|c|c|c|c|c|}
\hline \multirow[b]{2}{*}{ Methods } & \multicolumn{3}{|c|}{ Treatment Group } & \multicolumn{3}{|c|}{ Placebo } \\
\hline & \multicolumn{2}{|c|}{$\begin{array}{c}\text { Standardized Bias } \\
(1 / 2 \text { of } 95 \% \mathrm{CI})\end{array}$} & \multirow[t]{2}{*}{$\begin{array}{c}\text { Coverage } \\
\text { Probability }(\%)\end{array}$} & \multicolumn{2}{|c|}{$\begin{array}{c}\text { Standardized Bias } \\
(1 / 2 \text { of } 95 \% \mathrm{CI})\end{array}$} & \multirow[t]{2}{*}{$\begin{array}{c}\text { Coverage } \\
\text { Probability }(\%)\end{array}$} \\
\hline \multicolumn{5}{|c|}{ Missing mechanism: MCAR } & & \\
\hline BLS & 0.00 & $(0.15)$ & 95.0 & 0.01 & $(0.15)$ & 95.4 \\
\hline PMM & 0.00 & $(0.14)$ & 94.9 & -0.01 & $(0.14)$ & 94.1 \\
\hline PS & -0.01 & $(0.17)$ & 97.3 & 0.01 & $(0.17)$ & 97.0 \\
\hline MCMC & 0.00 & $(0.14)$ & 95.0 & -0.01 & $(0.14)$ & 94.9 \\
\hline $\mathrm{CC}$ & -0.01 & $(0.15)$ & 94.9 & -0.02 & $(0.15)$ & 95.1 \\
\hline LOCF & -0.35 & $(0.12)$ & 93.5 & -0.07 & $(0.12)$ & 94.8 \\
\hline \multicolumn{7}{|c|}{ Missing mechanism: MAR } \\
\hline BLS & 0.02 & $(0.16)$ & 95.7 & 0.02 & $(0.16)$ & 95.5 \\
\hline PMM & 0.86 & $(0.14)$ & 84.0 & 0.87 & $(0.14)$ & 85.3 \\
\hline PS & 0.71 & $(0.17)$ & 88.5 & 0.67 & $(0.17)$ & 91.4 \\
\hline MCMC & 0.04 & $(0.16)$ & 96.0 & 0.04 & $(0.16)$ & 95.5 \\
\hline $\mathrm{CC}$ & 0.17 & $(0.15)$ & 94.7 & 0.17 & $(0.15)$ & 95.7 \\
\hline LOCF & -1.48 & $(0.12)$ & 68.2 & -1.21 & $(0.12)$ & 78.3 \\
\hline
\end{tabular}

(d) MVN (Covariance: Toeplitz)

\begin{tabular}{|c|c|c|c|c|c|c|}
\hline \multirow[b]{2}{*}{ Methods } & \multicolumn{3}{|c|}{ Treatment Group } & \multicolumn{3}{|c|}{ Placebo } \\
\hline & \multicolumn{2}{|c|}{$\begin{array}{l}\text { Standardized Bias } \\
(1 / 2 \text { of } 95 \% \mathrm{CI})\end{array}$} & \multirow[t]{2}{*}{$\begin{array}{c}\text { Coverage } \\
\text { Probability }(\%)\end{array}$} & \multicolumn{2}{|c|}{$\begin{array}{l}\text { Standardized Bias } \\
(1 / 2 \text { of } 95 \% \mathrm{CI})\end{array}$} & \multirow[t]{2}{*}{$\begin{array}{c}\text { Coverage } \\
\text { Probability (\%) }\end{array}$} \\
\hline \multicolumn{5}{|c|}{ Missing mechanism: MCAR } & & \\
\hline $\mathrm{BLS}$ & 0.00 & $(0.17)$ & 93.8 & 0.01 & $(0.17)$ & 94.9 \\
\hline PMM & -0.02 & $(0.17)$ & 92.2 & 0.02 & $(0.17)$ & 94.9 \\
\hline PS & 0.00 & $(0.20)$ & 96.2 & 0.01 & $(0.20)$ & 96.6 \\
\hline MCMC & 0.01 & $(0.17)$ & 93.4 & 0.00 & $(0.17)$ & 95.1 \\
\hline $\mathrm{CC}$ & -0.03 & $(0.19)$ & 93.6 & -0.01 & $(0.19)$ & 95.1 \\
\hline LOCF & -0.30 & $(0.15)$ & 92.8 & -0.05 & $(0.15)$ & 95.4 \\
\hline \multicolumn{7}{|c|}{ Missing mechanism: MAR } \\
\hline $\mathrm{BLS}$ & 0.00 & $(0.18)$ & 93.0 & 0.00 & $(0.18)$ & 95.2 \\
\hline PMM & 0.86 & $(0.17)$ & 81.4 & 0.83 & $(0.17)$ & 85.3 \\
\hline PS & 0.77 & $(0.20)$ & 87.3 & 0.75 & $(0.20)$ & 88.8 \\
\hline $\mathrm{MCMC}$ & -0.01 & $(0.18)$ & 92.6 & 0.01 & $(0.18)$ & 94.3 \\
\hline $\mathrm{CC}$ & 0.38 & $(0.18)$ & 91.4 & 0.35 & $(0.18)$ & 94.0 \\
\hline LOCF & -1.45 & $(0.14)$ & 69.4 & -1.22 & $(0.14)$ & 79.1 \\
\hline
\end{tabular}

candidates in the stratum with high missing probability was too small due to the small sample size. On the other hand, the MVN-based methods (BLS and MCMC) had no bias. As shown in (6), completer analysis gave estimates with some bias under $R_{\text {Toeplitz }}$ where the correlation becomes smaller proportional to the distance between two visits.

\section{(2) Between-group difference in change}

Only LOCF had bias. The biases of the estimates by LOCF were less than $30 \%$ of the standard error. This result was the same as that obtained under MCAR.

\section{2) Coverage probability and interval length}

\section{(1) Within each treatment group}

LOCF gave shortest confidence intervals and precisions were misleadingly overestimated 
Table 2: Summary of the between-group difference in change from baseline at the final visit $(n=100 /$ group $)$

(a) Standardized bias and half length of $95 \% \mathrm{CI}$

\begin{tabular}{|c|c|c|c|c|c|c|c|c|}
\hline \multirow[b]{2}{*}{ Methods } & \multicolumn{2}{|c|}{ CS (Strong) } & \multicolumn{2}{|c|}{ CS (Weak) } & \multicolumn{2}{|c|}{$\mathrm{AR}(1)$} & \multicolumn{2}{|c|}{ Toeplitz } \\
\hline & \multicolumn{2}{|c|}{$\begin{array}{l}\text { Standardized Bias } \\
(1 / 2 \text { of } 95 \% \text { CI })\end{array}$} & \multicolumn{2}{|c|}{$\begin{array}{l}\text { Standardized Bias } \\
(1 / 2 \text { of } 95 \% \mathrm{CI})\end{array}$} & \multicolumn{2}{|c|}{$\begin{array}{l}\text { Standardized Bias } \\
(1 / 2 \text { of } 95 \% \mathrm{CI})\end{array}$} & \multicolumn{2}{|c|}{$\begin{array}{l}\text { Standardized Bias } \\
(1 / 2 \text { of } 95 \% \mathrm{CI})\end{array}$} \\
\hline \multicolumn{9}{|c|}{ Missing mechanism: MCAR } \\
\hline $\mathrm{BLS}$ & -0.01 & $(0.14)$ & 0.03 & $(0.27)$ & -0.01 & $(0.20)$ & -0.01 & $(0.24)$ \\
\hline PMM & 0.01 & $(0.13)$ & 0.02 & $(0.25)$ & 0.01 & $(0.19)$ & -0.02 & $(0.24)$ \\
\hline PS & 0.00 & $(0.21)$ & 0.02 & $(0.27)$ & 0.00 & $(0.24)$ & -0.02 & $(0.28)$ \\
\hline MCMC & 0.00 & $(0.14)$ & 0.01 & $(0.26)$ & 0.01 & $(0.20)$ & -0.01 & $(0.24)$ \\
\hline $\mathrm{CC}$ & 0.01 & $(0.14)$ & 0.00 & $(0.27)$ & 0.00 & $(0.21)$ & -0.01 & $(0.27)$ \\
\hline LOCF & -0.28 & $(0.11)$ & -0.14 & $(0.23)$ & -0.20 & $(0.16)$ & -0.17 & $(0.21)$ \\
\hline \multicolumn{9}{|c|}{ Missing mechanism: MAR } \\
\hline BLS & 0.02 & $(0.16)$ & 0.00 & $(0.33)$ & 0.01 & $(0.23)$ & -0.01 & $(0.26)$ \\
\hline PMM & 0.02 & $(0.14)$ & 0.02 & $(0.27)$ & 0.00 & $(0.20)$ & 0.00 & $(0.24)$ \\
\hline PS & 0.01 & $(0.18)$ & 0.03 & $(0.33)$ & 0.04 & $(0.23)$ & 0.00 & $(0.28)$ \\
\hline $\mathrm{MCMC}$ & -0.02 & $(0.16)$ & 0.02 & $(0.32)$ & 0.00 & $(0.23)$ & -0.01 & $(0.26)$ \\
\hline $\mathrm{CC}$ & 0.00 & $(0.14)$ & -0.01 & $(0.29)$ & 0.00 & $(0.21)$ & 0.02 & $(0.26)$ \\
\hline LOCF & -0.28 & $(0.12)$ & -0.14 & $(0.23)$ & -0.20 & $(0.16)$ & -0.16 & $(0.20)$ \\
\hline
\end{tabular}

(b) Coverage probability of $95 \% \mathrm{CI}$ for between-group difference in change from baseline at the final visit

\begin{tabular}{lcccc}
\hline \multirow{2}{*}{ Methods } & $\begin{array}{c}\text { CS (Strong) } \\
\text { Coverage } \\
\text { Probability (\%) }\end{array}$ & $\begin{array}{c}\text { CS (Weak) } \\
\text { Probability (\%) }\end{array}$ & $\begin{array}{c}\text { AR(1) } \\
\text { Probability (\%) }\end{array}$ & $\begin{array}{c}\text { Toeplitz } \\
\text { Poverage } \\
\text { Probability (\%) }\end{array}$ \\
\hline Missing mechanism: MCAR & & & & \\
\hdashline BLS & 95.2 & 94.2 & 94.5 & 94.7 \\
PMM & 93.6 & 93.2 & 93.4 & 93.6 \\
PS & 98.7 & 94.9 & 97.1 & 96.0 \\
MCMC & 95.2 & 95.1 & 94.4 & 95.1 \\
CC & 95.6 & 94.6 & 94.0 & 94.6 \\
LOCF & 94.8 & 94.0 & 94.1 & 93.5 \\
\hline Missing mechanism: & MAR & -1.5 & \\
\hline BLS & 95.5 & 95.0 & 94.5 & \\
PMM & 92.0 & 94.0 & 93.3 & 93.6 \\
PS & 94.5 & 93.9 & 93.3 & 93.8 \\
MCMC & 94.0 & 96.1 & 95.0 & 95.5 \\
CC & 94.8 & 95.0 & 93.7 & 94.4 \\
LOCF & 94.4 & 93.0 & 93.9 & 94.2 \\
\hline
\end{tabular}

under all covariance structures as was observed in MCAR. The actual coverage probabilities of BLS and MCMC were close to the nominal level and those of LOCF were lower than the nominal level by 15 to $80 \%$. In PMM, reductions of actual coverage probabilities were 10 to $15 \%$ except for compound symmetry with weak correlation. The largest reduction in PS was about $8 \%$. As shown in (6) the reduction in actual coverage probabilities in completer analysis was largest $(3 \%)$ under $R_{\text {Toeplitz }}$, and actual coverage probabilities were close to the nominal one under other covariance structures. The reason for this phenomenon is that the bias in the mean of actual values cancelled out by subtracting the baseline. 


\section{(2) Between-group difference in change}

LOCF also gave shortest confidence intervals in all methods. For actual coverage probabilities, less than 3\% reduction in PMM, less than $2 \%$ reduction in LOCF and less than $2 \%$ reduction in PS and completer were observed under four types of covariance structures. The actual coverage probabilities in BLS and MCMC, which are based on MVN, were close to the nominal one.

\section{Simulation study II: Finite mixture of MVN data}

\subsection{Simulation design}

In this section we consider a typical case of non-normal distribution that the population of interest consists of a mixture of two normal subpopulations. Our interest is to examine whether or not the MI procedures work well under such settings. The setting of the present simulation study is taken from an actual clinical trial for anti bronchial asthma drug. It is postulated here that the population of a new drug is a mixture of responders and nonresponders. The mean vectors in FEV1.0 for responders and non-responders are assumed to be

$$
E\left[\boldsymbol{Y}_{1 \_ \text {Res }}\right]=(2.50,3.00,3.30,3.50)^{T} \text { and } E\left[\boldsymbol{Y}_{1 \_ \text {NonRes }}\right]=(2.50,2.58,2.60,2.60)^{T},
$$

and standard deviations, which are the same across the four time points within each subpopulation, are 0.50 and 0.72 , respectively. The ratio of responders and non-responders is set to $3: 2$ and the total number of patients in the new drug group is set to 100 as in the previous simulations. Figure 4 shows the density functions of FEV1.0 value at visit 4 for responders, non-responders and the mixture of them. The mixture distribution is left-skewed. Covariance structures are assumed to be compound symmetry with strong correlation $(r=0.8)$ for both populations. As for placebo, the average response is assumed to be the same as that presented in section 5.1. The fraction of missing is also the same as the previous simulation (20\% at visit 3 and $30 \%$ at visit 4 ). The number of replications in the simulation is also 1000 .

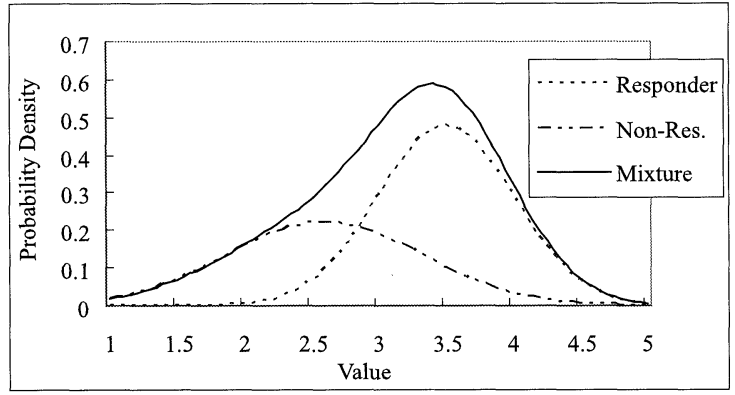

Fig. 4: A mixture distribution in FEV1.0 at Visit 4 of responders and non-responders

\subsection{Results}

The results for individual treatment groups are summarized for each missing mechanism in Table 3. The results of the between-group difference in change are summarized in Table 
Table 3: Standardized bias, half length of $95 \%$ CI and coverage probability for change from baseline at the final visit within treatment group ( $n=100$ / group)

\begin{tabular}{|c|c|c|c|c|c|c|}
\hline \multicolumn{7}{|c|}{ Mixture of two MVNs } \\
\hline \multirow[b]{2}{*}{ Methods } & \multicolumn{6}{|c|}{ Treatment Group } \\
\hline & \multicolumn{2}{|c|}{$\begin{array}{l}\text { Standardized Bias } \\
(1 / 2 \text { of } 95 \% \mathrm{CI})\end{array}$} & $\begin{array}{c}\text { Coverage } \\
\text { Probability }(\%)\end{array}$ & \multicolumn{2}{|c|}{$\begin{array}{c}\text { Standardized Bias } \\
(1 / 2 \text { of } 95 \% \mathrm{CI})\end{array}$} & $\begin{array}{c}\text { Coverage } \\
\text { Probability (\%) }\end{array}$ \\
\hline \multicolumn{4}{|c|}{ Missing mechanism: MCAR } & \multicolumn{3}{|c|}{ Missing mechanism: MAR } \\
\hline $\mathrm{BLS}$ & 0.02 & $(0.14)$ & 99.0 & -0.82 & $(0.15)$ & 92.4 \\
\hline PMM & 0.02 & $(0.13)$ & 98.8 & -0.14 & $(0.13)$ & 96.8 \\
\hline PS & 0.00 & $(0.18)$ & 99.5 & -0.04 & $(0.16)$ & 97.1 \\
\hline $\mathrm{MCMC}$ & 0.02 & $(0.14)$ & 99.1 & -0.81 & $(0.15)$ & 92.8 \\
\hline $\mathrm{CC}$ & 0.02 & $(0.14)$ & 98.5 & 0.03 & $(0.14)$ & 98.9 \\
\hline LOCF & -1.29 & $(0.11)$ & 80.8 & -2.18 & $(0.12)$ & 42.1 \\
\hline
\end{tabular}

Table 4: Standardized bias and coverage probability for between-group difference in change from baseline at the final visit $(n=100$ / group)

Mixture of two MVNs

\begin{tabular}{|c|c|c|c|c|c|c|}
\hline \multirow[b]{2}{*}{ Methods } & \multicolumn{6}{|c|}{ Between-group Difference } \\
\hline & \multicolumn{2}{|c|}{$\begin{array}{c}\text { Standardized Bias } \\
(1 / 2 \text { of } 95 \% \mathrm{CI})\end{array}$} & $\begin{array}{c}\text { Coverage } \\
\text { Probability (\%) }\end{array}$ & \multicolumn{2}{|c|}{$\begin{array}{c}\text { Standardized Bias } \\
(1 / 2 \text { of } 95 \% \text { CI })\end{array}$} & $\begin{array}{c}\text { Coverage } \\
\text { Probability (\%) }\end{array}$ \\
\hline \multicolumn{4}{|c|}{ Missing mechanism: MCAR } & \multicolumn{3}{|c|}{ Missing mechanism: MAR } \\
\hline BLS & 0.02 & $(0.17)$ & 98.1 & -0.74 & $(0.19)$ & 91.7 \\
\hline PMM & -0.02 & $(0.16)$ & 97.4 & -0.72 & $(0.16)$ & 89.2 \\
\hline PS & 0.01 & $(0.23)$ & 99.8 & -0.49 & $(0.21)$ & 93.7 \\
\hline $\mathrm{MCMC}$ & 0.00 & $(0.17)$ & 98.1 & -0.74 & $(0.19)$ & 92.6 \\
\hline $\mathrm{CC}$ & 0.00 & $(0.17)$ & 97.9 & 0.01 & $(0.17)$ & 97.6 \\
\hline LOCF & -0.97 & $(0.14)$ & $87: 4$ & -0.97 & $(0.14)$ & 87.1 \\
\hline
\end{tabular}

4. The simulation results are outlined below.

a) MCAR

1) Standardized bias of mean change

(1) Within-group change

Only LOCF had bias in the change from baseline and the bias of the estimate by LOCF was less than 1.3 times its standard error.

(2) Between-group difference in change

Only LOCF had bias and the bias of the estimate by LOCF was less than $97 \%$ of the standard error.

\section{2) Coverage probability and interval length}

(1) Within-group change

The length of confidence interval of LOCF was also the shortest and less than $15 \%$ reduction in actual coverage from the nominal level was observed. The actual coverage probabilities of other statistical methods were greater than the nominal value. 


\section{(2) Between-group difference in change}

LOCF gave the shortest interval in the all methods and about $8 \%$ reduction in actual coverage from the nominal level was observed. In other statistical methods, actual coverage was greater than the nominal one.

b) MAR

1) Standardized bias of mean change

(1) Within each treatment group

The bias of estimates by LOCF was the largest in all methods, which was about 2 times relative to its standard error. The estimates by MVN-based methods (BLS and MCMC) had bias, which were about $80 \%$ of standard error. The biases by matching-based methods (PMM and PS) were smaller than those by MVN-based methods.

\section{(2) Between-group difference in change}

The statistical methods except for the completer analysis had bias. The biases in BLS, MCMC and PMM were about $70 \%$ of their standard errors. The bias in PS and LOCF were about $50 \%$ and $100 \%$ of SE respectively. Completer analysis had no bias.

\section{2) Coverage probability and interval length}

\section{(1) Within each treatment group}

The actual coverage probabilities in BLS and MCMC based on MVN were lower than the nominal level by $2 \%$. The actual coverage probabilities in completer analysis, PMM and PS were close to the nominal value.

\section{(2) Between-group difference in change}

The length of $95 \%$ confidence interval in LOCF was the shortest. The actual coverage probabilities in MI based on MVN (BLS and MCMC) were lower than the nominal by $3 \%$. And 5\% reduction in coverage of PMM was observed. One reason for this seems to be the normality of measurements in the placebo group. On the other hand, the actual coverage of PS was better than that of MI based on MVN by 1 to $2 \%$. About $8 \%$ reduction in coverage from nominal level in LOCF was observed.

\section{Concluding remarks}

This paper evaluated the performance of statistical methods for analysis of incomplete data in the context of longitudinal clinical trials with relatively small sample size $(n=100)$. Four types of imputation methods in MI were compared with completer analysis and LOCF. In section 4 , the biases in the estimates of the change from baseline based on completer analysis and LOCF under censored multivariate normal distribution were mathematically derived. In section 5, Monte Carlo simulations were conducted, whose settings are based on the results of FEV1.0 in an actual clinical trial. Performance of the statistical methods was evaluated in the cases of multivariate normal data and also of non-normal data (a finite mixture of responders and non-responders).

In summary, the amount of bias and the reduction in actual coverage probabilities were smaller in the case of inference for the between-group difference in change compared with that for the within-group change. This is due to the fact that biases associated with two group estimates are often in the same direction and the biases cancel out when betweengroup difference is calculated. Even in cases of small sample, multiple imputation based on MVN (BLS, MCMC) performed the best for the inference for both within-group change and 
between-group difference in change under MVN data with various covariance structures. Because BLS and MCMC are essentially the same algorithms under monotone missing pattern, both methods gave similar results. Performance of completer analysis was also satisfactory except for within-group change in the case of Toeplitz type covariance. On the other hand LOCF gave the largest biases in estimates, shortest confidence intervals and largest reduction in actual coverage probabilities under many situations. These findings suggest that LOCF has potential difficulties which may draw misleadingly biased inferences. In case of small samples the use of PMM and PS, which are matching-based methods, should be performed with care because of the bias in estimates and of reduction of the actual coverage probabilities of confidence intervals. This is due to the fact that it is difficult to find matching candidates who have truly close values for missing observations with a small sample. In the case of non-normal data, 2 to $3 \%$ reductions in actual coverage probabilities compared with the nominal level were observed in MVN-based methods (BLS and MCMC). On the other hand reduction in coverage in PS was about 1 to $2 \%$ lower compared with MVN-based methods. This is because the PS is a nonparametric method and produces longer confidence intervals.

In the case of small sample, performance of MI highly depends on the imputation methods. Therefore, when researchers use MI it is important to check whether the assumption of MAR and underlying assumptions for each imputation method are plausible. The convergence of MCMC should also be checked carefully using diagnostic tools (e.g. time-series plots for parameter estimates). In addition, it would also be important to collect information after patient's dropout and to include almost all relevant variables into the imputation model. We did not examine the methods to deal with missing data under NMAR (Not Missing At Random). This should be a problem that needs further investigation in the future.

\section{Acknowledgements}

The authors would like to thank the editors and two referees for their many helpful comments and suggestions on this paper. This research was partly supported by the Grantin-Aid for Scientific Research (A) No. 16200022.

\section{REFERENCES}

Abe, T., Inaba, Y. and Iwasaki, M. (2005). Comparison of software and statistical analysis methods for incomplete data. Bulletin of the Computational Statistics of Japan 18, 7994. (in Japanese)

Allison, P. D. (2000). Multiple imputation for missing data. A cautionary tale. Sociological Methods \& Research 28, 301-309.

Barnard, J. and Rubin, D. B. (1999). Small-sample degrees of freedom with multiple imputation. Biometrika 86, 948-955.

Barnes, S. A., Lindborg, S. R. and Seaman Jr., J. W. (2006). Multiple imputation techniques in small sample clinical trials. Statistics in Medicine 25, 233-245.

Dempster, A. P., Laird, N. M., and Rubin, D. B. (1977). Maximum likelihood from incomplete data via the EM algorithm. Journal of the Royal Statistical Society, Series B 39, 1-38 (with discussion).

Heitjan, F. and Little, R. J. A. (1991). Multiple imputation for the fatal accident reporting system. Applied Statistics 40, 13-29. 
Horton, N. J. and Lipsitz, S. R. (2001). Multiple imputation in practice: comparison of software packages for regression models with missing variables. American Statistician $55,244-254$.

Iwasaki, M. (2002a). Statistical Analysis with Incomplete Data. Tokyo: Economist-sha. (in Japanese)

Iwasaki, M. (2002b). Analysis of pretest-posttest data and the regression to the mean: a review. Japanese Journal of Behaviormetrics 29, 247-273. (in Japanese)

Johnson, N. L. and Kotz, S. (1970). Distributions in Statistics. Continuous Univariate Distributions-1. New York: John Wiley \& Sons.

Johnson, N. L. and Kotz, S. (1972). Distributions in Statistics. Continuous Multivariate Distributions. New York: John Wiley \& Sons.

Lavori, P. W., Dawson, R. and Shera, D. (1995). A multiple imputation strategy for clinical trial with truncation of patient data. Statistics in Medicine 14, 1913-1925.

Little, R. J. A. and Rubin, D. B. (2002). Statistical Analysis with Missing Data, 2nd Edition. New York: John Wiley \& Sons.

Liu, G. and Gould L. (2002). Comparison of alternative strategies for analysis of longitudinal trials with dropouts. Journal of Biopharmaceutical Statistics 12, 207-226.

Reiss T. F., Chervinsky P., Dockhorn R. J., Shingo S., Seidenberg B. and Edwards T. B. (1998). Montelukast, a once-daily leukotriene receptor antagonist, in the treatment of chronic asthma. Archives of Internal Medicine 158, 1213-1220.

Rosenbaum, P. R. and Rubin, D. B. (1983). The central role of the propensity score in observational studies for causal effects. Biometrika 70, 41-55.

Rubin, D. B. (1976). Inference and missing data. Biometrika 63, 581-592.

Rubin, D. B. (1987). Multiple Imputation for Nonresponse in Surveys. New York: John Wiley \& Sons.

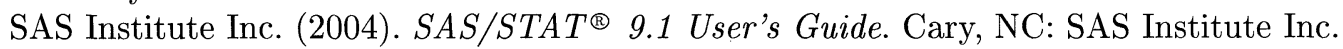

Schafer, J. L. (1997). Analysis of Incomplete Multivariate Data. London: Chapman \& Hall.

Tang, L., Song, J., Belin, T. R. and Unützer, J. (2005). A comparison of imputation methods in a longitudinal randomized clinical trial. Statistics in Medicine 24, 2111-2128.

Tanner, M. A. and Wong, W. H. (1987). The calculation of posterior distribution by data augmentation. Journal of the American Statistical Association 82, 528-540 (with discussion).

Watanabe, M. and Yamaguchi, K. (eds.) (2000). The EM Algorithm and Issues Related to Incomplete Data. Tokyo: Taga-Publishing. (in Japanese)

Watanabe, M. and Yamaguchi, K. (eds.) (2004). The EM Algorithm and Related Statistical Models. New York: Marcel Dekker.

(Received: August 25, 2006, Accepted: March 1, 2007) 